\title{
Reciprocal regulation of $m$ TOR complexes in pancreatic islets from humans with type 2 diabetes
}

\author{
Ting Yuan ${ }^{1} \cdot$ Sahar Rafizadeh ${ }^{1} \cdot$ Kanaka Durga Devi Gorrepati $^{1} \cdot$ Blaz Lupse $^{1}$ • \\ Jose Oberholzer $^{2} \cdot$ Kathrin Maedler $^{1,3}$ - Amin Ardestani ${ }^{1}$
}

Received: 13 October 2016 / Accepted: 2 December 2016/Published online: 21 December 2016

(C) Springer-Verlag Berlin Heidelberg 2016

\begin{abstract}
Aims/hypothesis Mechanistic target of rapamycin complex 1 (mTORC1) is a master regulator of nutritional status at the cellular and organismic level. While mTORC1 mediates beta cell growth and expansion, its hyperactivation has been observed in pancreatic islets from animal models of type 2 diabetes and leads to beta cell loss. We sought to determine whether such mTORC1 activation occurs in humans with type 2 diabetes or in metabolically stressed human islets and whether mTORC1 blockade can restore beta cell function of diabetic islets.

Methods Human islets isolated from non-diabetic controls and individuals with type 2 diabetes, as well as human islets and INS-1E cells exposed to increased glucose $(22.2 \mathrm{mmol} / \mathrm{l})$,
\end{abstract}

Ting Yuan, Sahar Rafizadeh and Kanaka Durga Devi Gorrepati are joint first authors.

Kathrin Maedler and Amin Ardestani are joint senior authors.

Electronic supplementary material The online version of this article (doi:10.1007/s00125-016-4188-9) contains peer-reviewed but unedited supplementary material, which is available to authorised users.

Kathrin Maedler

kmaedler@uni-bremen.de

$\triangle$ Amin Ardestani

ardestani.amin@gmail.com

1 Islet Biology Laboratory, Centre for Biomolecular Interactions Bremen, University of Bremen, Leobener Straße NW2, Room B2080, 28359 Bremen, Germany

2 Division of Transplantation, University of Illinois at Chicago, Chicago, IL, USA

3 German Center for Diabetes Research (DZD) project partner, University of Bremen, Bremen, Germany were examined for mTORC1/2 activity by western blotting analysis of phosphorylation of mTORC1 downstream targets ribosomal protein S6 kinase 1 (S6K1), S6 and eukaryotic translation initiation factor $4 \mathrm{E}$ binding protein 1 (4E-BP1) and mTORC2 downstream targets Akt and N-myc downstream regulated 1 (NDRG1). mTORC1/2 complexes' integrity was assessed by immunoprecipitation and subsequent western blot analysis. Cell-type specific expression of activated mTORC1 in human islets was examined by immunostaining of pS6 (Ser 235/236) in human islet sections. Beta cell function was measured by glucose-stimulated insulin secretion (GSIS).

Results While mTORC2 signalling was diminished, mTORC1 activity was markedly increased in islets from patients with type 2 diabetes and in islets and beta cells exposed to increased glucose concentrations. Under high-glucose conditions in metabolically stressed human islets, we identified a reciprocal regulation of different mTOR complexes, with functional upregulation of $\mathrm{mTORC} 1$ and downregulation of mTORC2. pS6 immunostaining showed beta cell-specific upregulation of $\mathrm{mTORC} 1$ in islets isolated from patients with type 2 diabetes. Inhibition of mTORC1-S6K1 signalling improved GSIS and restored mTORC2 activity in islets from patients with type 2 diabetes as well as in islets isolated from diabetic $d b / d b$ mice and mice fed a high-fat/high-sucrose diet. Conclusions/interpretation Our data show the aberrant mTORC1 activity in islets from patients with type 2 diabetes, in human islets cultured under diabetes-associated increased glucose conditions and in diabetic mouse islets. This suggests that elevated $\mathrm{mTORC} 1$ activation is a striking pathogenic hallmark of islets in type 2 diabetes, contributing to impaired beta cell function and survival in the presence of metabolic stress.

Keywords Beta cells · Glucose - Human islets - mTORC1 · mTORC2 2 Nutrients $\cdot$ Type 2 diabetes 


$\begin{array}{ll}\text { Abbreviations } \\ \text { 4E-BP1 } & \text { eIF4E-binding protein 1 } \\ \text { eIF4E } & \text { Eukaryotic translation initiation factor 4E } \\ \text { GSIS } & \text { Glucose-stimulated insulin secretion } \\ \text { HFD } & \text { High-fat/high-sucrose diet } \\ \text { mTOR } & \text { Mechanistic target of rapamycin } \\ \text { mTORC1 } & \text { mTOR complex-1 } \\ \text { mTORC2 } & \text { mTOR complex-2 } \\ \text { NDRG1 } & \text { N-myc downstream regulated 1 } \\ \text { S6K1 } & \text { Ribosomal protein S6 kinase 1 } \\ \text { siRNA } & \text { Small interfering RNA }\end{array}$

\section{Introduction}

Pancreatic beta cells play a central role in controlling glucose homeostasis in response to metabolic fluctuations. The beta cells must coordinate multiple cellular metabolic processes to enable their insulin secretory responses to adapt to nutrient availability. Type 2 diabetes manifests itself by a progressive decline in beta cell functional adaptation and ultimate loss of beta cell mass by apoptosis [1]. The compensatory insulin response by pancreatic beta cells is a key rate-limiting step determining whether at-risk obese individuals develop diabetes. Nutrient overload, such as chronically increased glucose and NEFA concentrations, has been proposed as a main underlying pathological factor leading to beta cell deterioration during progression of type 2 diabetes [2]. Identifying the signalling mechanisms by which nutrient overload leads to impaired insulin action and beta cell death is instrumental for better understanding of the molecular changes underlying functional loss of beta cell mass and development of therapeutic strategies for the treatment of type 2 diabetes.

The mechanistic target of rapamycin (mTOR) is a master regulator of diverse cellular functions, such as metabolism, proliferation and survival, forming at least two functionally distinct complexes, mTOR complex-1 (mTORC1) and mTOR complex-2 (mTORC2). mTORC1 phosphorylates various substrates, such as eukaryotic translation initiation factor $4 \mathrm{E}$ (eIF4E)-binding protein 1 (4E-BP1), S6 kinase 1 (S6K1) and SREBP, to promote anabolic processes and mTORC2 phosphorylates AGC kinases, including Akt and serum and glucocorticoid-regulated kinase 1 (SGK1), to enhance cell proliferation and survival. mTORC1 is a key nutrient sensor, integrating diverse extra- and intra-cellular cues to downstream signalling pathways in response to growth factors, stress, nutrient availability and other stimuli [3]. Considering the importance of metabolic control and mTORC1's role as a key sensor and regulator of cellular energy, it is not surprising that this signalling is dysregulated in a variety of disease states such as cancer and type 2 diabetes [3, 4]. mTORC1 activity is highly upregulated in the liver, fat, muscle and pancreatic islets of obese and high-fat-fed rodents [5-8]; this correlates with elevated circulating levels of insulin, pro-inflammatory cytokines and nutrients such as glucose and NEFAs and may represent a common consequential mechanism triggered by these driving forces in obesity. mTORC1 activity is constitutively elevated under conditions of nutrient satiation in the rodent beta cell line in vitro [9] and in the pancreatic islets of type 2 diabetic mice [8]. We therefore asked in the present study whether mTORC1 activity is altered in human isolated islets under conditions of metabolic stress and diabetes and whether acute inhibition of mTORC1 signalling can improve beta cell function.

\section{Methods}

Human islet isolation, cell culture and treatment Human islets were isolated from pancreases of non-diabetic organ donors and from patients with type 2 diabetes at the University of Illinois at Chicago, Geneva University, Leiden University, Lille University and at ProdoLabs (Table 1) and cultured on extracellular matrix (ECM) coated dishes (Novamed, Jerusalem, Israel) as described previously [10]. The clonal rat beta cell line INS-1E was kindly provided by C. Wollheim, University of Geneva, Switzerland and Lund University, Sweden. Human islets were cultured in complete CMRL-1066 (Invitrogen, Carlsbad, CA, USA) medium at $5.5 \mathrm{mmol} / \mathrm{l}$ glucose and INS-1E cells were cultured in complete RPMI-1640 medium at $11.1 \mathrm{mmol} / \mathrm{l}$ glucose and were exposed chronically to increased glucose at $22.2 \mathrm{mmol} / 1$. In some experiments, human islets were additionally cultured with $10 \mu \mathrm{mol} / 1 \mathrm{~S} 6 \mathrm{~K} 1$ selective inhibitor PF-4708671 (Calbiochem, San Diego, CA, USA). Ethical approval for the use of human islets was granted by the Ethics Committee of the University of Bremen. INS-1E cells were routinely tested for mycoplasma contamination and were negative.

Animals, islet isolation and culture Islets were isolated from high-fat/high-sucrose diet (HFD)-treated mice, leptinreceptor-deficient $L e p r^{\mathrm{db} / \mathrm{db}}(d b / d b)$ mice and non-diabetic heterozygous Lepr $^{\mathrm{db} /+}(d b /+)$ mice. Islets were cultured overnight in RPMI 1640 medium and treated with or without S6K1 selective inhibitor PF-4708671 for $4 \mathrm{~h}$ following protein isolation or glucose-stimulated insulin secretion (GSIS). See electronic supplementary material [ESM] Methods for details.

Transfections An adapted improved transfection protocol including $\mathrm{Ca}^{2+}-\mathrm{KRH}$ medium was used to knock down raptor expression in human islets as described in detail in ESM Methods.

Western blot analysis Human islets and INS-1E cells were washed in ice-cold PBS and lysed in RIPA lysis buffer. Protein concentrations were determined with the BCA protein assay (Pierce, Thermo Fisher Scientific, Waltham, MA, USA). 
Table 1 Human islet donor characteristics

\begin{tabular}{|c|c|c|c|c|c|c|}
\hline Donor & Age (years) & $\begin{array}{l}\mathrm{BMI} \\
\left(\mathrm{kg} / \mathrm{m}^{2}\right)\end{array}$ & Sex & $\mathrm{HbA}_{1 \mathrm{c}}(\%)$ & $\begin{array}{l}\mathrm{HbA}_{1 \mathrm{c}} \\
(\mathrm{mmol} / \mathrm{mol})\end{array}$ & Cause of death \\
\hline \multirow{11}{*}{$\begin{array}{l}\text { Type } 2 \\
\text { diabetes }\end{array}$} & 37 & 39.3 & $\mathrm{~F}$ & 7.3 & 57 & Anoxia \\
\hline & 48 & 41 & M & 6.5 & 48 & Unknown \\
\hline & 56 & 24.3 & M & 5.1 & 32 & Stroke \\
\hline & 54 & 29 & M & 6.5 & 48 & Vascular/hypertension \\
\hline & 57 & 36.3 & $\mathrm{~F}$ & 8.2 & 66 & Cerebrovascular/stroke \\
\hline & 48 & 30.2 & M & 7.9 & 63 & Trauma \\
\hline & 56 & 40 & $\mathrm{~F}$ & 8.1 & 65 & Cerebrovascular/stroke \\
\hline & 59 & 29.2 & $\mathrm{~F}$ & 7.4 & 57 & Anoxia, CPR \\
\hline & 51 & 24.4 & M & 6.9 & 52 & Stroke \\
\hline & 58 & 39.3 & M & 8.9 & 74 & Anoxia \\
\hline & 61 & 28 & M & 5.2 & 33 & Cerebrovascular \\
\hline Mean & 53.2 & 32.9 & & & & \\
\hline SEM & 1.9 & 1.8 & & & & \\
\hline \multirow[t]{13}{*}{ Controls } & 23 & 24.5 & $\mathrm{~F}$ & 4.9 & 30 & Head trauma \\
\hline & 51 & 24.4 & $\mathrm{~F}$ & $<6$ & $<42$ & Head trauma \\
\hline & 31 & 23.8 & M & $<6$ & $<42$ & Suicide (gunshot) \\
\hline & 48 & 25 & M & $<6$ & $<42$ & Stroke \\
\hline & 54 & 37.3 & $\mathrm{~F}$ & 4.9 & 30 & Meningitis \\
\hline & 51 & 27.5 & M & $<6$ & $<42$ & Unknown \\
\hline & 26 & 46.6 & M & 4.8 & 29 & Head trauma \\
\hline & 56 & 33.1 & $\mathrm{~F}$ & $<6$ & $<42$ & Cerebrovascular/stroke \\
\hline & 82 & 22.2 & $\mathrm{~F}$ & $<6$ & $<42$ & Vascular event \\
\hline & 64 & 27.8 & M & 5.2 & 33 & Stroke \\
\hline & 57 & 23.1 & $\mathrm{~F}$ & $<6$ & $<42$ & Stroke \\
\hline & 38 & 34 & $\mathrm{~F}$ & 5 & 31 & Anoxia \\
\hline & 51 & 24.1 & M & $<6$ & $<42$ & Stroke \\
\hline Mean & 47.8 & 28.0 & & & & \\
\hline SEM & 4.3 & 1.8 & & & & \\
\hline
\end{tabular}

CPR, cardiopulmonary resuscitation; $\mathrm{F}$, female; $\mathrm{M}$, male
Equivalent amounts of protein from each treatment group were run on a NuPAGE 4-12\% Bis-Tris gel (Invitrogen) and electrically transferred onto polyvinylidene difluoride membranes. See ESM Methods for details.

Co-immunoprecipitation Immunoprecipitation was performed as described in detail in ESM Methods. Briefly, $500 \mu \mathrm{g}$ of total lysate was incubated with rabbit anti-mTOR on a rotator at $4{ }^{\circ} \mathrm{C}$ overnight. Immunocomplexes were then captured with Protein A Agarose Fast Flow (Millipore, Temecula, CA, USA) and were re-suspended in sample buffer and separated by NuPAGE 4-12\% Bis-Tris gels (Invitrogen).

Immunofluorescence After isolation, human islets were processed for staining as previously described [11]. Sections (4 $\mu \mathrm{m})$ were deparaffinised, rehydrated and incubated with primary antibodies overnight at $4{ }^{\circ} \mathrm{C}$, followed by FITCconjugated secondary antibody (1:100; Jackson
ImmunoResearch Laboratories, West Grove, PA, USA) for $1 \mathrm{~h}$ at RT. Slides were mounted with Vectashield with DAPI (Vector Labs, Burlingame, CA, USA). See ESM Methods for details.

GSIS For acute insulin release in response to glucose, primary islets from humans and mice were washed and pre-incubated (30 min) in $\mathrm{KRB}$ containing $2.8 \mathrm{mmol} / 1$ glucose and $0.5 \%$ BSA. KRB was then replaced by KRB-2.8 mmol/1 glucose for $1 \mathrm{~h}$ (basal), followed by an additional $1 \mathrm{~h}$ in KRB$16.7 \mathrm{mmol} / \mathrm{l}$ glucose (stimulated). Total protein content was extracted with RIPA buffer. Insulin was determined using human insulin ELISA (ALPCO Diagnostics, Salem, NH, USA). Secreted and content insulin was normalised to total protein content.

Statistical analysis The experiments were not randomised and the investigators were not blinded to allocation during 
experiments and outcome assessment. All values were expressed as means \pm SEM with the number of independent individual experiments (biological replicates) presented in the figure legends. The different groups were compared by unpaired two-tail Student's $t$ test; $p<0.05$ was considered statistically significant.

\section{Results}

mTORC1 is hyperactivated in diabetic islets To examine the pathological relevance of mTORC1 activation in type 2 diabetes, we investigated whether mTORC1 activity was altered in islets isolated from patients with type 2 diabetes. Activation of mTORC1 was detected by phosphorylation analysis of its downstream targets S6K1 at Thr 389 (pS6K), the direct S6K substrate ribosomal protein S6 at Ser 235/236 (pS6) and 4E-BP1 at Thr 37/46 (p4E-BP1) (Fig. 1a). Western blots of pS6 and p4E-BP1 from human islets showed that mTORC1 activity was elevated in the islets from patients with type 2 diabetes (Fig. 1b). To thoroughly examine the activation of mTORC1 in control and diabetic islets, we analysed pS6 and p4E-BP1 signals by quantitative densitometry of immunoblots from ten non-diabetic and eight diabetic individuals (see Table 1). Our data showed that the mTORC1 activity was significantly increased in islets isolated from individuals with type 2 diabetes compared with the controls (1.76-fold increase in pS6; 3.07 -fold increase in $\mathrm{p} 4 \mathrm{E}-\mathrm{BP} 1 ; p<0.05$; Fig. 1c). To mimic a type 2 diabetes-like condition, islets isolated from humans were chronically cultured in elevated glucose concentrations for $72 \mathrm{~h}$. Diabetes-associated elevated glucose profoundly upregulated mTORC1 activity in the islets (Fig. 1d, e), as demonstrated by increased phosphorylation of S6K1, S6 and 4E-BP1. Together, these data show that a diabetic milieu robustly upregulates mTORC1 activity in human islets.

Pancreatic beta cells are the source of upregulated mTORC1 in islets from individuals with type 2 diabetes As pancreatic islets are a composite of many cell types, including insulin-expressing beta cells and glucagon-expressing alpha cells, we aimed to determine whether increased mTORC1 activity is present in beta or non-beta cells. Celltype-specific expression of activated mTORC1 in human islets was examined by immunostaining of pS6 (Ser 235/ 236). Immunofluorescent labelling of pS6 in combination
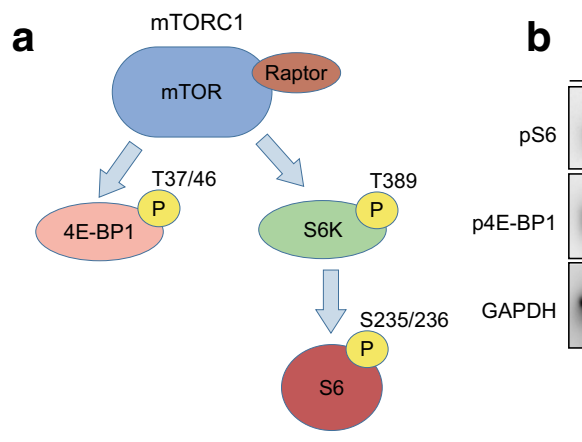

b

d

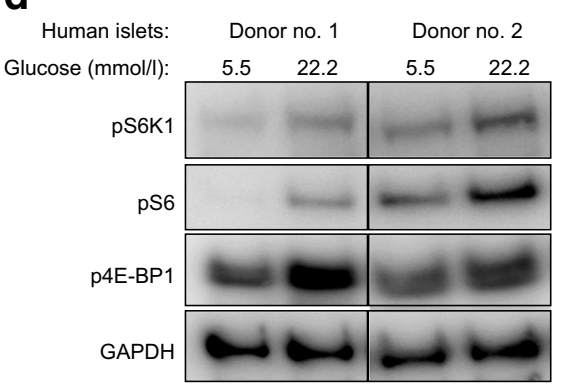

Fig. 1 mTORC1 is hyperactivated in diabetic islets. (a) Scheme depicting downstream targets of mTORC1. Activation of mTORC1 leads to phosphorylation and inactivation of 4E-BP1 (pT37/46) and activation of S6K (pT389). Active S6K phosphorylates its downstream target ribosomal protein S6 (pS235/236). (b, c) Human islets isolated from nondiabetic controls $(n=10)$ and individuals with type 2 diabetes (T2D) $(n=8)$ were analysed for mTORC1 activity. A representative western blot is shown (b) and quantitative densitometry analysis of pS6 and p4E-BP1 signals was performed (c). White bars, control islets; grey bars, islets from
C

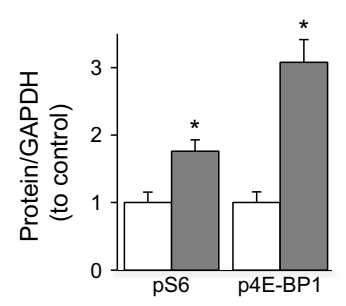

\section{e}
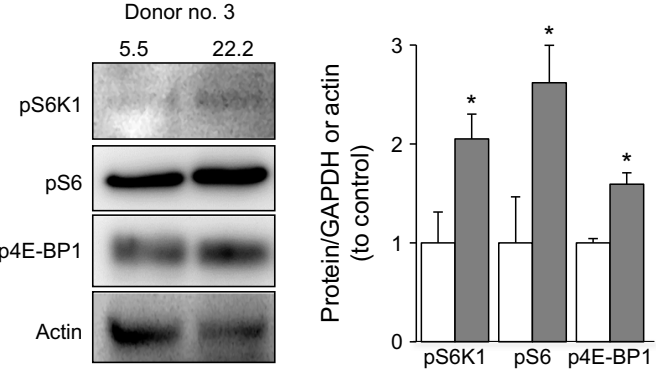

individuals with T2D. (d, e) Human islets were treated with $22.2 \mathrm{mmol} / \mathrm{l}$ glucose for 3 days. Representative western blots (d) and quantitative densitometry analysis (e) of pS6K, pS6 and p4E-BP1 signals from three non-diabetic controls $(n=3)$ are shown. White bars, control human islets; grey bars, high-glucose-treated human islets. Data are pooled from three independent experiments $(n=3)$. Actin/GAPDH was used as loading control for western blots. Data show means \pm SEM. $* p<0.05$ compared with non-diabetic controls (c) or $5.5 \mathrm{mmol} / 1$ glucose-treated control human islets (e) 
with insulin or glucagon revealed higher mTORC1 activity in islets from individuals with type 2 diabetes. Activated S6 was present predominantly in insulin-expressing beta cells (Fig. 2a, c) and expression levels were very low in glucagon-expressing alpha cells (Fig. 2b, c). Quantification of insulin- or glucagon-co-expressing pS6 in sections of islets isolated from multiple human non-diabetic controls and individuals with type 2 diabetes (T2D) showed significant upregulation of activated S6 in beta cells and thus confirms higher mTORC1 activity in diabetic islets, indeed in the beta cell with insignificant expression in alpha cells (Fig. 2c). mTORC1 activity was also analysed in the established beta cell line INS-1E to provide comparison with primary human islets in terms of response to chronically elevated glucose concentrations. Prolonged culture of INS-1E cells with elevated glucose concentrations $(22.2 \mathrm{mmol} / \mathrm{l})$ led to robust upregulation of mTORC1, as represented by increased phosphorylation of S6K1, S6 and 4E-BP1 (Fig. 2d, e), confirming that mTORC1 hyperactivation indeed occurs in the beta cell.

\section{Reciprocal regulation of different mTOR complexes in di-} abetic islets The activity and functional status of the two different mTOR complexes were examined by immunoprecipitation of endogenous mTOR and subsequent analysis of the mTOR-associated proteins raptor (representing mTORC1 activity) and rictor (representing mTORC2 activity). While endogenous raptor co-precipitated with mTOR (raptormTOR complex) was highly increased and confirmed higher activity of mTORC1 under high-glucose conditions, rictor co-precipitated with mTOR (rictor-mTOR complex) was drastically reduced upon prolonged culture of human islets with high glucose suggesting a reciprocal regulation of the two mTOR complexes in metabolically stressed human islets (Fig. 3a-c).

To confirm the functional decline of mTORC2 in diabetic islets, we investigated whether mTORC2 activity was changed in islets isolated from patients with type 2 diabetes. Activation of mTORC2 was assessed by phosphorylation analysis of its downstream targets Akt at Ser 473 (pAkt) and $\mathrm{N}$-myc downstream regulated 1 (NDRG1) at Thr 346 (pNDRG1) (Fig. 4a). Consistent with the lower integrity of mTORC2 under high-glucose conditions, western blot analysis of pAkt and pNDRG1 from human islets showed that mTORC2 activity was diminished in the islets from patients with type 2 diabetes (Fig. 4b). To thoroughly examine the activation of mTORC2 in control and diabetic islets, we analysed pAkt and pNDRG1 signals by quantitative densitometry of immunoblots from eight non-diabetic and seven diabetic individuals. Our data showed that mTORC2 activity was significantly reduced in the diabetic islets compared with non-diabetic controls (77\% and 53\% reduction in pAkt and pNDRG1, respectively, $p<0.05$; Fig. $4 \mathrm{c}$ ).
mTORC1-S6K1 inhibition improves insulin secretion in islets from individuals with type 2 diabetes mTORC1 upregulation correlates with beta cell dysfunction in islets from individuals with type 2 diabetes $[12,13]$. Knockdown of the critical component of mTORC1, raptor, is sufficient to increase insulin secretion in rodent beta cell lines [14]. Therefore, we hypothesised that mTORC1 inhibition would improve beta cell function in already-diabetic islets in the ex vivo setting. To date, no selective mTORC1 inhibitor has been discovered. Although rapamycin has widely been used to block mTORC1, it also inhibits mTORC2 [15-17]. Furthermore, the effects of rapamycin on islet biology are complex and controversial, with many laboratories reporting detrimental effects of rapamycin on beta cell function and survival [18]. Nevertheless, we tested the effect of different concentrations of rapamycin on islets from individuals with type 2 diabetes. Rapamycin at concentrations of 10 or $20 \mathrm{nmol} / \mathrm{l}$ had no effect on GSIS in diabetic islets (data not shown). This, as well as previous results, makes rapamycin an inappropriate approach by which to target mTORC1 in human islets.

As S6K1 is one of the major downstream effectors of mTORC1, we tested PF-4708671, a novel cell-permeable piperazinyl pyrimidine compound recently characterised as a selective S6K1 inhibitor [19], as an alternative approach for targeting mTORC1. The efficiency of PF-4708671 at blocking S6K1 signalling was confirmed in multiple human isolated islet preparations (Fig. 5c). Islets isolated from nondiabetic control individuals and from patients with type 2 diabetes were treated with $10 \mu \mathrm{mol} / \mathrm{l}$ PF-4708671 for $4 \mathrm{~h}$. In line with previously published observations [20], the insulin content was drastically reduced in islets from individuals with type 2 diabetes when compared with non-diabetic controls (ESM Fig. 1). After treatment of non-diabetic and diabetic islets with S6K1 inhibitor for $4 \mathrm{~h}$, there was no change in insulin content (ESM Fig. 1). While short-term treatment with inhibitor had no effect on GSIS in islets from non-diabetic individuals (Fig. 5a), it improved glucose-stimulated insulin response by 2.3 -fold (with a strong tendency towards statistical significance $[p=0.05]$ ), compared with the response in non-treated islets from individuals with type 2 diabetes. Such functional restoration occurred independently in all tested batches of human islets isolated from three patients with type 2 diabetes (Fig. 5b), indicating the beneficial effects of mTORC1-S6K1 signalling blockade.

While mTORC2 is critical for maintaining beta cell function [21], chronic hyperactivation of mTORC1 diminishes mTORC2-Akt signalling through multiple negativefeedback loops [22-24]. Thus, we examined whether mTORC 1 inhibition can restore depleted mTORC 2 in diabetic islets. In line with our insulin secretion data, S6K1 inhibitor increased mTORC2 activity as shown by increased phosphorylation of Akt (demonstrated independently in islets isolated from three donors with type 2 diabetes) (Fig. $5 \mathrm{c}, \mathrm{d}$ ). This 

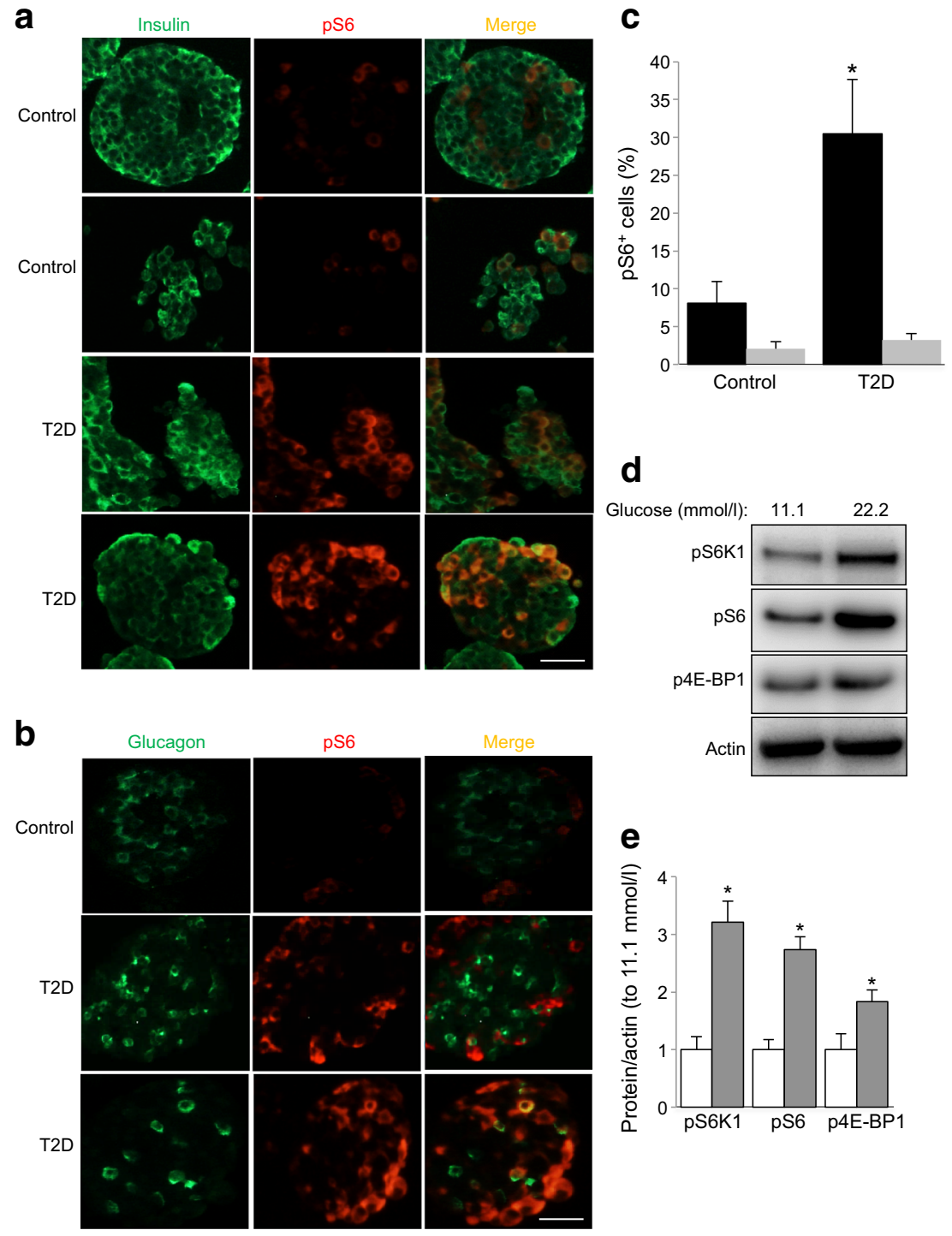

Fig. 2 mTORC1 is hyperactivated in diabetic beta cells. (a, b) Freshly isolated human islets from non-diabetic individuals as well as from patients with type 2 diabetes (T2D) were cultured overnight. Fixed paraffinembedded islet sections were double-stained for pS6 (red) and insulin (green) (a) or glucagon (green) (b). Representative images from two non-diabetic control islets and two diabetic islets are shown. Scale bars, $100 \mu \mathrm{m}$. (c) Quantitative analysis of insulin- or glucagon-co-expressing pS6 in human islet sections from control and diabetic individuals. Data are means \pm SEM from three non-diabetic controls $(n=3)$ and three individuals with T2D $(n=3)$. The number of cells that were counted for controls and T2D donors, respectively, was 2700 and 1725 insulin-

suggests that successful inhibition of the mTORC1-S6K1 axis improved mTORC2 function most probably by restraining mTORC1-mediated negative-feedback loops.

To further corroborate the detrimental impact of hyperactivated $\mathrm{mTORC} 1$ on beta cell function in diabetic islets, we selectively inhibited mTORC1 by targeting its central component raptor. Targeted inhibition of endogenous mTORC1 by small interfering RNA (siRNA)-mediated silencing of raptor resulted in substantially increased GSIS positive beta cells and 1493 and 1585 glucagon-positive alpha cells. Black bars, pS6-insulin co-positive cells; grey bars, pS6-glucagon copositive cells. ${ }^{*} p<0.05$ compared with non-diabetic controls. (d, e) INS-1E cells were treated with $22.2 \mathrm{mmol} / 1$ glucose for 2 days. Representative western blots (d) and quantitative densitometry analysis of pS6K, pS6 and p4E-BP1 signals (e) are shown. Data are means \pm SEM from three independent experiments $(n=3)$. White bars, control INS-1E cells; grey bars, high-glucose-treated INS-1E cells. Actin was used as loading control. ${ }^{*} p<0.05$ compared with $11.1 \mathrm{mmol} / 1$ glucose-treated control INS-1E cells

(Fig. 5e, f) and efficiently reduced mTORC1 signalling (Fig. 5g), confirming the inhibitory action of upregulated human islet mTORC1 in type 2 diabetes.

S6K1 inhibition improves insulin secretion in isolated islets from obese diabetic mice A progressive decline of beta cell function leading to beta cell exhaustion, loss of compensatory beta cell adaptation, glucose intolerance and type 2 diabetes in response to long-term HFD have 
a Human islet donor no. 1
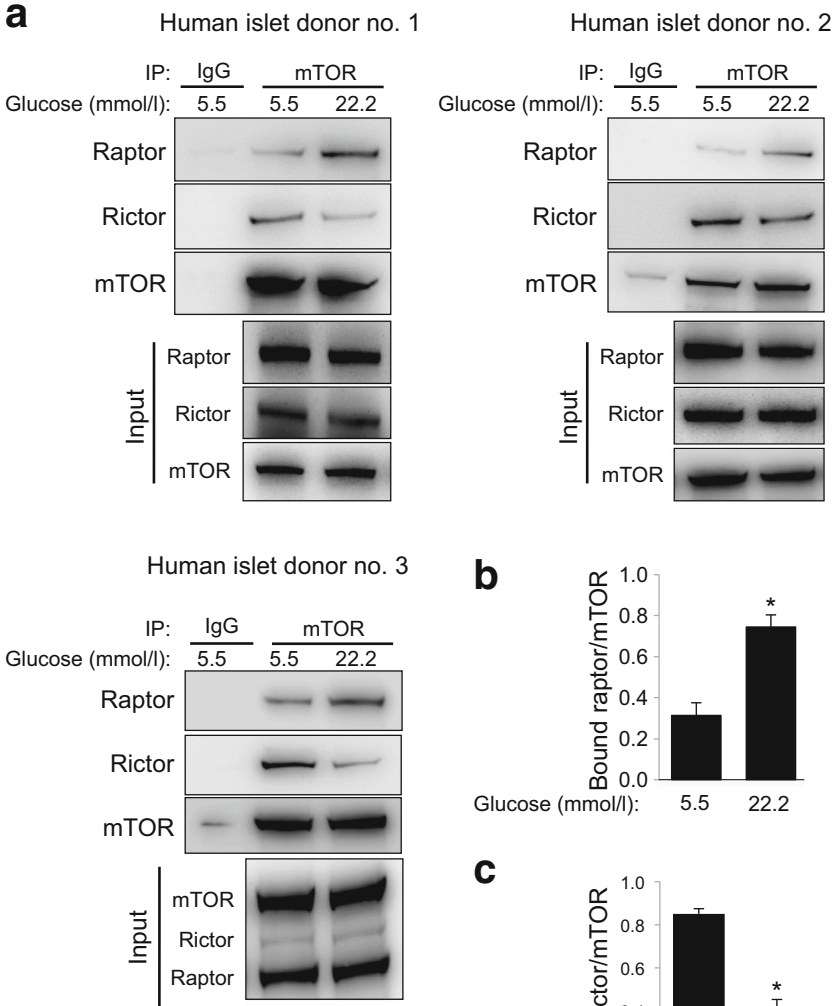

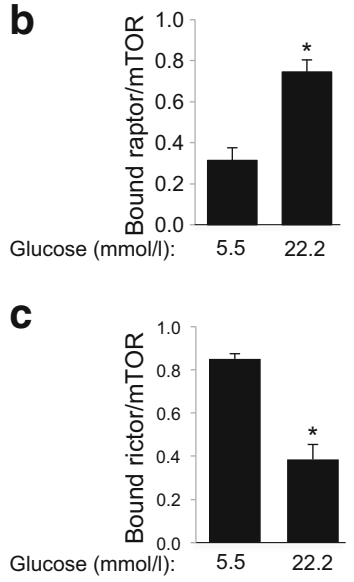

Glucose $(\mathrm{mmol} / \mathrm{l}): \quad 5.5 \quad 22.2$

Fig. 3 Reciprocal regulation of mTORC1/2 complex integrity by chronically elevated glucose in human islets. (a) Human islets were treated with $22.2 \mathrm{mmol} / \mathrm{l}$ glucose for 3 days. Co-immunoprecipitations (IP) were performed using anti-IgG and anti-mTOR antibodies and western blots of precipitates and input fractions were analysed for raptor, rictor and mTOR. 'Input' is the total islet protein lysate before immunoprecipitation. $(\mathbf{b}, \mathbf{c})$ Pooled quantitative densitometry analysis of co-precipitated raptor (b) or rictor (c) with $\mathrm{mTOR}$ from three independent experiments from three different human islet donors $(n=3)$. Data show means \pm SEM. ${ }^{*} p<0.05$ compared with $5.5 \mathrm{mmol} / \mathrm{l}$ glucose-treated control human islets

been clearly established in mice [10]. To further confirm the beneficial effect of mTORC1-S6K1 blockade on beta cell function, we examined whether acute S6K1 inhibition could improve insulin secretion in islets isolated from mice that had been treated with HFD for 17 weeks. Consistent with the improved beta cell function of human diabetic islets upon S6K1 inhibition, the diabetic mouse islets treated with S6K1 inhibitor showed significantly higher GSIS response compared with untreated HFD islets (Fig. 6a); the stimulatory index was highly improved (Fig. 6b). Intriguingly, and in line with human islet data, S6K1 inhibitor treatment greatly increased mTORC2 activity, as demonstrated by phosphorylation of Akt and NDRG1 (Fig. 6c, d). The efficiency of S6K1 inhibition in restoring mTORC2 activity was also tested in isolated islets from leptin- receptor-deficient $d b / d b$ mice as another mouse model of type 2 diabetes. Inverse regulation of different mTOR complexes, with higher mTORC1 activity and lower mTORC2 activity, was reproduced in pancreatic islets of diabetic $d b / d b$ mice (Fig. 6e). This confirms previously published observations in $d b / d b$ mouse islets [8][25]. S6K1 inhibitor treatment fully blocked S6 phosphorylation and normalised $\mathrm{mTORC} 2$ activity in isolated $d b / d b$ islets (Fig. 6e), confirming mTORC1-S6K1-mediated mTORC2 depletion as a possible pathogenic signalling hallmark of diabetic islets.

\section{Discussion}

The beta cell's loss of response to chronically elevated nutrients and resultant deficit in function and mass represents one of the most fundamental pathological hallmarks of type 2 diabetes. Despite the finding of constitutive mTORC1 activity in the pancreatic islets of mouse models of type 2 diabetes [8], hyperactivity of mTORC1 was unknown in human islets in type 2 diabetes so far. This study provides the first direct evidence that islets from individuals with type 2 diabetes, as well as metabolically stressed human islets, display an opposite regulation of mTORC $1 / 2$ signals, with higher mTORC1 and lower mTORC2 activity. Inhibition of mTORC1 signalling either through S6K1 inhibition or through knockdown of raptor improved insulin secretion in human and mouse diabetic islets. This observation provides a mechanism for the collapse of the beta cell's ability to adapt function and mass in response to excess nutrients during obesity. Consequently, type 2 diabetes develops with hyperglycaemia and relative insulin deficiency.

Pancreatic beta cell-specific Tsc2-knockout mice, in which mTORC1 is constitutively active, show a biphasic pattern of beta cell turnover $[8,26]$. While sustained mTORC1 activation leads to hyperinsulinaemia and improved glucose homeostasis through enhanced beta cell hypertrophy and hyperplasia in young mice, prolonged constitutive mTORC1 hyperactivation diminishes pancreatic beta cell mass by inducing apoptosis, leading to progressive hyperglycaemia and the development of diabetes in older mice. This cell-autonomous biphasic regulatory pattern of mTORC1 function can explain, at least in part, signalling alterations in the widely accepted model of beta cell deterioration 'compensation/decompensation switch' during the progression of type 2 diabetes. In the initial functional compensatory phase, metabolic drivers such as insulin resistance and nutrient excess increase beta cell mTORC1 activity; this correlates with higher insulin production as well as compensatory beta cell hypertrophy and hyperplasia, suggesting mTORC1 as a key positive 
regulator of beta cell function and mass [27]. However, in the subsequent detrimental decompensatory phase, chronic activation of mTORC1 caused by sustained nutrient overload (mainly high glucose and NEFA) leads to beta cell exhaustion, functional collapse and ultimate cell death. The higher mTORC1 activity detected in the islets of individuals with type 2 diabetes in our study is consistent with observations made in animal models of type 2 diabetes and may be the consequence of nutrient overload in pancreatic beta cells.

How does chronic activation of mTORC1 promote impaired beta cell function and survival? It has been firmly established that mTORC1 mediates potent negativefeedback loops that restrain upstream mitogenic signalling from insulin/IGF receptor pathways [22-24]. These loops cause long-term compensatory mechanisms inhibiting Akt kinase via multiple mechanisms (e.g. mTORC1-S6K suppresses Akt signalling through phosphorylation of IRS1/2, Grb10 and Sin1 proteins [22-24]). Notably, chronic mTOR activation negatively regulates beta cell survival by direct IRS2 phosphorylation and subsequent proteasomal degradation [24]. Consistently, rapamycin-mediated mTORC1 inhibition upregulates IRS2 and Akt phosphorylation in mouse islets in vivo and in vitro, suggesting the presence of the negative functional loop from mTORC1 that prevents IRS2-Akt signalling [28]. A novel regulatory negativefeedback loop was established through mTORC1-S6K-mediated phosphorylation of the mTORC2 subunit Sin1 [23], inhibiting the mTORC2-Akt signalling axis, which is essential for maintaining normal beta cell mass [21]. This may explain the defective Akt-Ser473 phosphorylation (site of mTORC2 phosphorylation) seen in stressed beta cells $[10,25]$ and in islets from individuals with type 2 diabetes in our study and may be associated with the impaired mTORC2 complex integrity under prolonged glucose stimulation as presented here. Akt is the master prosurvival kinase in beta cells $[10,21,27,29]$. One major detrimental outcome of the negative-feedback loops initiated by constitutive mTORC1 hyperactivity would be impaired Akt-mediated pro-proliferative and pro-survival responses. Indeed, defective Akt signalling is a hallmark of diabetic beta cells in the context of type 2 diabetes [10,25]. Using various ex vivo experimental models of diabetes, including islets isolated from patients with type 2 diabetes as well as from hyperglycaemic HFD-treated and $d b / d b$ mice, we demonstrated that inhibition of mTORC1-S6K1 signalling leads to striking restoration of mTORC2 activity; this suggests the existence of functional inhibitory loop(s) from mTORC1 to mTORC2. We speculate that such negative-feedback mechanisms triggered by mTORC1S6K1 hyperactivation may operate in vivo in beta cells in type 2 diabetes. The identification of specific pathway(s) downstream of activated mTORC1, involved in the decline of mTORC2 signalling, may provide a better understanding of such complex interplay and some clues to its reversal.

Another so far unexplored but important mechanistic link between mTORC1 activity and beta cell failure in diabetes is the impairment of autophagy. Defective autophagy has been implicated in the process of beta cell failure in type 2 diabetes [26, 30, 31] and it is well established that mTORC1 hyperactivity results in inhibition of autophagy [26]. Mice deficient in autophagy-promoting protein 7 (Atg7), an essential component of autophagy, show exacerbated development of diabetes under high-fat diet, with a

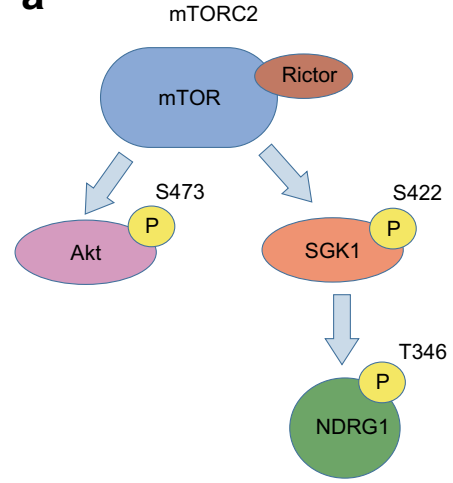

b

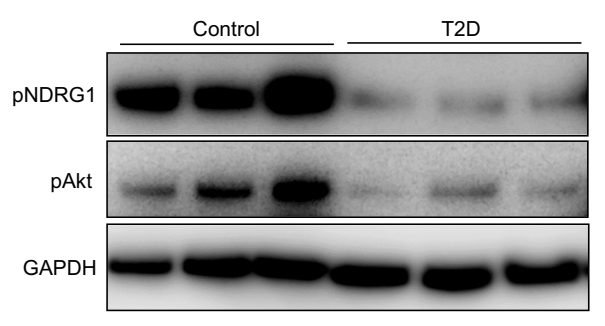

C

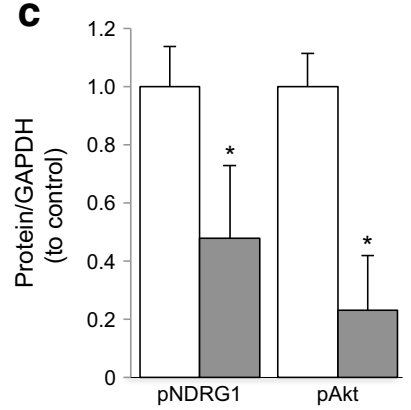

Fig. 4 mTORC2 activity is diminished in diabetic islets. (a) Scheme depicting downstream targets of mTORC2. Activation of mTORC2 leads to phosphorylation and activation of Akt (pS473) and SGK1 (pS422). Active SGK1 phosphorylates its downstream target NDRG1 (pT346). (b, c) Human islets isolated from eight non-diabetic controls $(n=8)$ and seven individuals with type 2 diabetes (T2D) $(n=7)$ were analysed for
mTORC2 activity. pNDRG1 and pAkt were analysed by western blotting. Representative western blot (b) and quantitative densitometry analysis (c) of pNDRG1 and pAkt signals. GAPDH was used as loading control. White bars, control islets; grey bars, T2D islets. Data show means \pm SEM. $* p<0.05$ compared with non-diabetic controls 
a

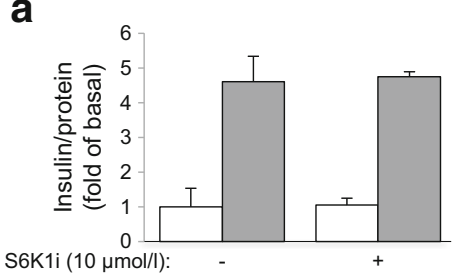

b

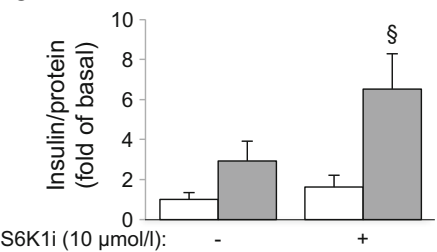

C
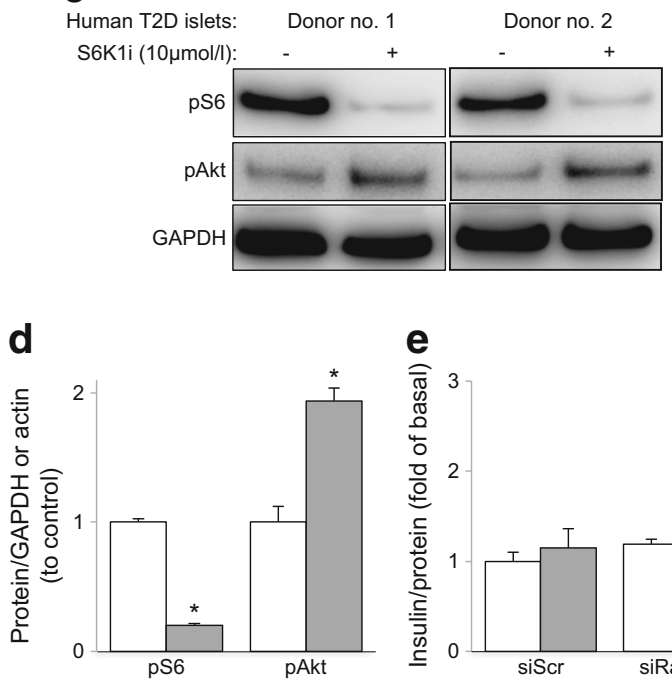

e

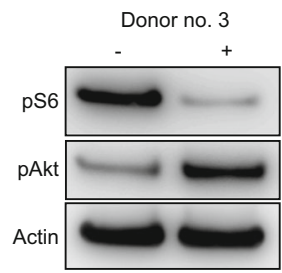

$\mathbf{f}$
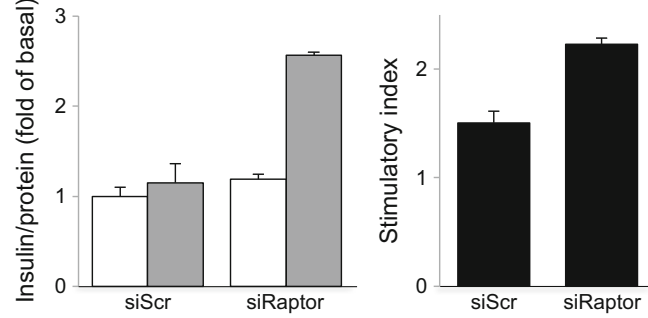

g

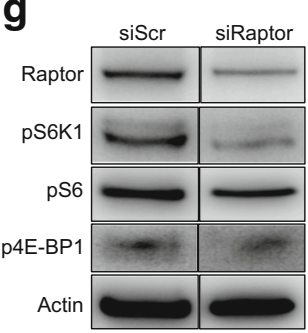

Fig. 5 S6K1 inhibition improves insulin secretion in islets from patients with type 2 diabetes. (a, b) Isolated islets from non-diabetic individuals (three donors; twelve to sixteen technical replicates per group) (a) and patients with type 2 diabetes (T2D) (three donors; eight to ten technical replicates per group) (b) were left untreated or treated with $10 \mu \mathrm{mol} / \mathrm{l} \mathrm{PF}-$ 4708671 (S6K1 inhibitor; S6K1i) for $4 \mathrm{~h}$. Insulin secretion during $1 \mathrm{~h}$ incubation with $2.8 \mathrm{mmol} / \mathrm{l}$ (basal) and $16.7 \mathrm{mmol} / \mathrm{l}$ glucose (stimulated) was measured, normalised to protein content. White bars, basal insulin; grey bars, stimulated insulin. (c, d) Human islets isolated from three individuals with T2D $(n=3)$ were analysed for $\mathrm{pS} 6$ and pAkt. Western blots for three different human islets donors (c) and quantitative densitometry analysis of pS6 and pAkt signals (d) are shown. White bars, untreated human islets; grey bars, S6K1 inhibitor-treated human islets.

diminished pancreatic beta cell mass and function [30]. Intriguingly, islets in type 2 diabetes accumulate autophagic vacuoles and autophagosomes concomitant with an increase in apoptotic beta cell death [32], suggesting an impaired flux of autophagy. In type 2 diabetes, the aberrant activation of $\mathrm{mTORC} 1$ in islets may provide a unique (e-g) Islets isolated from patients with T2D (two donors; six technical replicates per group) were transfected with control (non-targeting) siRNA (siScr) or raptor siRNA (siRaptor). Insulin secretion during $1 \mathrm{~h}$ incubation with $2.8 \mathrm{mmol} / \mathrm{l}$ (basal) and $16.7 \mathrm{mmol} / \mathrm{l}$ glucose (stimulated) was measured (e), normalised to protein content. White bars, basal insulin; grey bars, stimulated insulin. The insulin stimulatory index (f) denotes the ratio of insulin secreted in response to $16.7 \mathrm{mmol} / \mathrm{l}$ to that secreted in response to $2.8 \mathrm{mmol} / \mathrm{l}$ glucose. Representative western blots of raptor, pS6K, pS6 and p4E-BP1 are shown (g). Both lanes were run on the same gel but were non-contiguous. Data show means \pm SEM. ${ }^{\S} p=0.05$ compared with S6K1 inhibitor-treated basal (b); * $p<0.05$ compared with untreated T2D islets (d)

explanation for the chronic deterioration of the physiologically protective autophagy pathway during beta cell failure.

Despite the prevailing interest in mTORC1 inhibition for type 2 diabetes therapy, studies using mTOR inhibitors such as rapamycin show controversial results. While rapamycin 

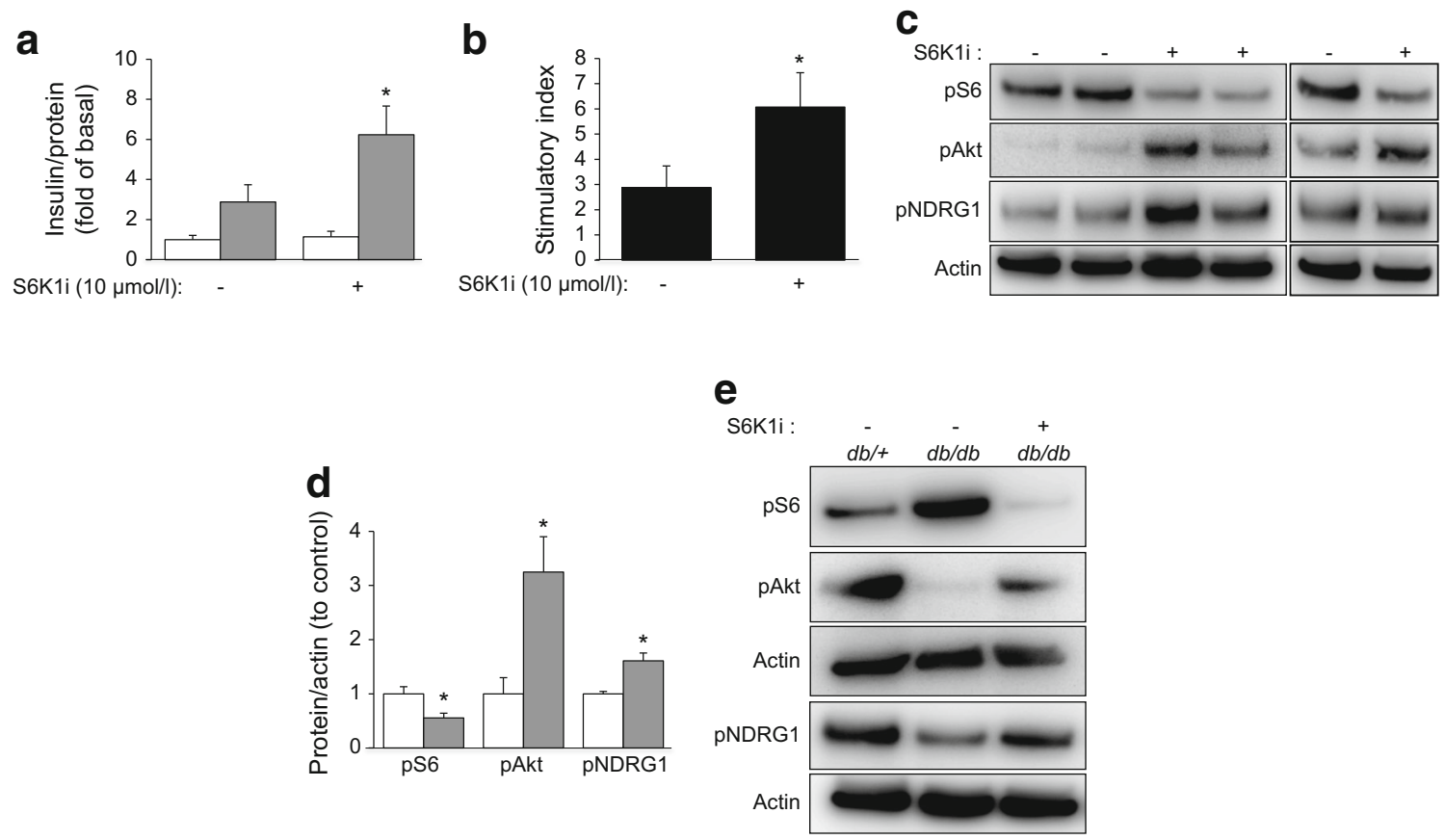

Fig. 6 S6K1 inhibitor improves insulin secretion in islets from diabetic mice. (a-d) Islets isolated from $\mathrm{C} 57 \mathrm{BL} / 6 \mathrm{~J}$ mice that had been treated with HFD for 17 weeks were cultured overnight and then treated with or without $10 \mu \mathrm{mol} / 1 \mathrm{PF}-4708671$ (S6K1 inhibitor; S6K1i) for $4 \mathrm{~h}$. Insulin secretion during $1 \mathrm{~h}$ incubation with $2.8 \mathrm{mmol} / \mathrm{l}$ (basal) and $16.7 \mathrm{mmol} / \mathrm{l}$ (stimulated) glucose was measured (a), normalised to protein content. The insulin stimulatory index (b) denotes the ratio of insulin secreted in response to $16.7 \mathrm{mmol} / 1$ to that secreted in response to $2.8 \mathrm{mmol} / 1$ glucose ( $n=8$ per group). The isolated islets were analysed for $\mathrm{pS6}$, pAkt and

suppresses stress-induced apoptosis in beta cell lines [9, 33] and improves beta cell function and thus corrects glucose homeostasis in the Akita mouse model of type 2 diabetes [34], chronic inhibition of mTORC1 by rapamycin causes glucose intolerance in mice $[17,28]$. This is due to the fact that chronic inhibition of $\mathrm{mTORC} 1$ with rapamycin disrupts mTORC2, which is crucial for the insulin-mediated suppression of hepatic gluconeogenesis [17] and for maintaining pancreatic functional beta cell mass [21]. Alternatively, our data show that blocking mTORC1-S6K1 signalling using a highly specific S6K1 inhibitor enhances insulin secretion in islets from individuals with type 2 diabetes and from a mouse model of type 2 diabetes ex vivo, suggesting that the elevated mTORC 1 activity seen in our study negatively regulates beta cell function. Interestingly, Shum et al [35] reported that rapamycin treatment compromised glucose metabolism and failed to improve Akt phosphorylation in liver, fat and muscle of HFD-treated mice and that, in contrast, S6K1 inhibition by PF-4708671 improved glucose tolerance and corrected HFD-induced impaired Akt phosphorylation in metabolically active tissues of obese mice. These previous data together with our study in human islets suggest the mTORC1-S6K1 axis as a potential therapeutic target for treatment of type 2 diabetes.

pNDRG1. Representative western blots of three different isolations (c) and subsequent quantitative densitometry analysis of pS6, pAkt and pNDRG1 signals (d) $(n=3)$ are shown. White bars, untreated HFD islets; grey bars, S6K1 inhibitor-treated HFD islets. (e) Islets isolated from diabetic 12-week-old $d b / d b$ mice and their heterozygous non-diabetic littermate controls $(d b /+)$ were left untreated or were treated with S6K1 inhibitor for $4 \mathrm{~h}$. Representative western blots of pS6, pAkt and pNDRG1 are shown. Data show means \pm SEM. $* p<0.05$ compared with untreated HFD islets

Acknowledgements Human islets were provided through the integrated islet distribution program (IIDP) supported by NIH and JDRF and the ECIT Islet for Basic Research program supported by JDRF (JDRF award 31-2008-413). We thank J. Kerr-Conte and F. Pattou (European Genomic Institute for Diabetes, Lille, France) for human islet isolations and $\mathrm{K}$. Hennekens (University of Bremen) for the excellent technical assistance. Some of the data were presented as an abstract at the 52nd EASD Annual Meeting in Munich in 2016.

Data availability All data generated or analysed during this study are included in this published article (and its supplementary information file).

Funding This work was supported by the Chinese Scholarship Council (CSC), the German Research Foundation (DFG), the European Research Council (ERC), JDRF and the German Diabetes Center grant (DZD) from the BMBF.

Duality of interest The authors declare that there is no duality of interest associated with this manuscript.

Contribution statement AA conceived the project. TY, SR, KG, BL, $\mathrm{AA}$ and $\mathrm{KM}$ designed and performed experiments and analysed data. JO contributed reagents or analytical tools and contributed to interpretation of data. AA and KM wrote the paper and supervised the project. All authors critically reviewed the manuscript for important intellectual content and approved the final version to be published. AA and $\mathrm{KM}$ are the guarantors of this work. 


\section{References}

1. Butler AE, Janson J, Bonner-Weir S, Ritzel R, Rizza RA, Butler PC (2003) Beta-cell deficit and increased beta-cell apoptosis in humans with type 2 diabetes. Diabetes 52:102-110

2. Robertson RP, Harmon J, Tran PO, Poitout V (2004) Beta-cell glucose toxicity, lipotoxicity, and chronic oxidative stress in type 2 diabetes. Diabetes 53(Suppl 1):S119-S124

3. Laplante M, Sabatini DM (2012) mTOR signaling in growth control and disease. Cell 149:274-293

4. Zoncu R, Efeyan A, Sabatini DM (2011) mTOR: from growth signal integration to cancer, diabetes and ageing. Nat Rev Mol Cell Biol 12:21-35

5. Um SH, Frigerio F, Watanabe M et al (2004) Absence of S6K1 protects against age- and diet-induced obesity while enhancing insulin sensitivity. Nature 431:200-205

6. Khamzina L, Veilleux A, Bergeron S, Marette A (2005) Increased activation of the mammalian target of rapamycin pathway in liver and skeletal muscle of obese rats: possible involvement in obesitylinked insulin resistance. Endocrinology 146:1473-1481

7. Tremblay F, Brule S, Hee Um S et al (2007) Identification of IRS-1 Ser-1101 as a target of S6K1 in nutrient- and obesity-induced insulin resistance. Proc Natl Acad Sci U S A 104:14056-14061

8. Shigeyama Y, Kobayashi T, Kido Y et al (2008) Biphasic response of pancreatic beta-cell mass to ablation of tuberous sclerosis complex 2 in mice. Mol Cell Biol 28:2971-2979

9. Bachar E, Ariav Y, Ketzinel-Gilad M, Cerasi E, Kaiser N, Leibowitz G (2009) Glucose amplifies fatty acid-induced endoplasmic reticulum stress in pancreatic beta-cells via activation of mTORC1. PLoS One 4, e4954

10. Ardestani A, Paroni F, Azizi Z et al (2014) MST1 is a key regulator of beta cell apoptosis and dysfunction in diabetes. Nat Med 20:385397

11. Sauter NS, Schulthess FT, Galasso R, Castellani LW, Maedler K (2008) The antiinflammatory cytokine interleukin-1 receptor antagonist protects from high-fat diet-induced hyperglycemia. Endocrinology 149:2208-2218

12. Del Guerra S, Lupi R, Marselli L et al (2005) Functional and molecular defects of pancreatic islets in human type 2 diabetes. Diabetes 54:727-735

13. Ohtsubo K, Chen MZ, Olefsky JM, Marth JD (2011) Pathway to diabetes through attenuation of pancreatic beta cell glycosylation and glucose transport. Nat Med 17:1067-1075

14. Le Bacquer O, Queniat G, Gmyr V, Kerr-Conte J, Lefebvre B, Pattou F (2013) mTORC1 and mTORC2 regulate insulin secretion through Akt in INS-1 cells. J Endocrinol 216:21-29

15. Sarbassov DD, Ali SM, Sengupta S et al (2006) Prolonged rapamycin treatment inhibits mTORC2 assembly and Akt/PKB. Mol Cell 22:159-168

16. Schreiber KH, Ortiz D, Academia EC, Anies AC, Liao CY, Kennedy BK (2015) Rapamycin-mediated mTORC2 inhibition is determined by the relative expression of FK506-binding proteins. Aging Cell 14:265-273

17. Lamming DW, Ye L, Katajisto P et al (2012) Rapamycin-induced insulin resistance is mediated by mTORC2 loss and uncoupled from longevity. Science 335:1638-1643

18. Barlow AD, Nicholson ML, Herbert TP (2013) Evidence for rapamycin toxicity in pancreatic beta-cells and a review of the underlying molecular mechanisms. Diabetes 62:2674-2682
19. Pearce LR, Alton GR, Richter DT et al (2010) Characterization of PF-4708671, a novel and highly specific inhibitor of p70 ribosomal S6 kinase (S6K1). Biochem J 431:245-255

20. Marchetti P, Del Guerra S, Marselli L et al (2004) Pancreatic islets from type 2 diabetic patients have functional defects and increased apoptosis that are ameliorated by metformin. J Clin Endocrinol Metab 89:5535-5541

21. Gu Y, Lindner J, Kumar A, Yuan W, Magnuson MA (2011) Rictor/ mTORC2 is essential for maintaining a balance between beta-cell proliferation and cell size. Diabetes 60:827-837

22. Efeyan A, Sabatini DM (2010) mTOR and cancer: many loops in one pathway. Curr Opin Cell Biol 22:169-176

23. Liu P, Gan W, Inuzuka H et al (2013) Sin1 phosphorylation impairs mTORC2 complex integrity and inhibits downstream Akt signalling to suppress tumorigenesis. Nat Cell Biol 15:1340-1350

24. Briaud I, Dickson LM, Lingohr MK, McCuaig JF, Lawrence JC, Rhodes CJ (2005) Insulin receptor substrate-2 proteasomal degradation mediated by a mammalian target of rapamycin (mTOR)induced negative feedback down-regulates protein kinase Bmediated signaling pathway in beta-cells. J Biol Chem 280:22822293

25. Wang L, Liu Y, Yan Lu S et al (2010) Deletion of Pten in pancreatic ss-cells protects against deficient ss-cell mass and function in mouse models of type 2 diabetes. Diabetes 59:3117-3126

26. Bartolome A, Kimura-Koyanagi M, Asahara S et al (2014) Pancreatic beta-cell failure mediated by mTORC1 hyperactivity and autophagic impairment. Diabetes 63:2996-3008

27. Blandino-Rosano M, Chen AY, Scheys JO et al (2012) mTORC1 signaling and regulation of pancreatic beta-cell mass. Cell cycle (Georgetown, Tex) 11:1892-1902

28. Fraenkel M, Ketzinel-Gilad M, Ariav Y et al (2008) mTOR inhibition by rapamycin prevents beta-cell adaptation to hyperglycemia and exacerbates the metabolic state in type 2 diabetes. Diabetes 57 : 945-957

29. Ardestani A, Maedler K (2016) MST1: a promising therapeutic target to restore functional beta cell mass in diabetes. Diabetologia 59:1843-1849

30. Jung HS, Chung KW, Won Kim J et al (2008) Loss of autophagy diminishes pancreatic beta cell mass and function with resultant hyperglycemia. Cell Metab 8:318-324

31. Stienstra R, Haim Y, Riahi Y, Netea M, Rudich A, Leibowitz G (2014) Autophagy in adipose tissue and the beta cell: implications for obesity and diabetes. Diabetologia 57:1505-1516

32. Masini M, Bugliani M, Lupi R et al (2009) Autophagy in ?human type 2 diabetes pancreatic beta cells. Diabetologia 52: $1083-1086$

33. Farrelly AM, Kilbride SM, Bonner C, Prehn JH, Byrne MM (2011) Rapamycin protects against dominant negative-HNF1A-induced apoptosis in INS-1 cells. Apoptosis: Int J Program Cell Death 16: $1128-1137$

34. Bachar-Wikstrom E, Wikstrom JD, Ariav Y et al (2013) Stimulation of autophagy improves endoplasmic reticulum stress-induced diabetes. Diabetes 62:1227-1237

35. Shum M, Bellmann K, St-Pierre P, Marette A (2016) Pharmacological inhibition of S6K1 increases glucose metabolism and Akt signalling in vitro and in diet-induced obese mice. Diabetologia 59:592-603 